\title{
Precision level measurement based on time-domain reflection (TDR) measurements
}

\author{
M. Gerding, T. Musch, and B. Schiek
}

RF and Microwave Engineering Institute Ruhr-University Bochum, Ruhr-Universität Bochum, Universitätsstrasse 150, 44780 Bochum, Germany

\begin{abstract}
A system for a high precision multi target level measurement based on guided microwave pulses is presented.

A wide-band technique based on time-domain reflectometry (TDR) in combination with a TEM-waveguide as the probe fulfils the requirements of mm-precision level measurements in tanks. The coaxial waveguide provides very low dispersion for wide-band signals. Inside the coaxial waveguide the different fluids with their specific dielectric constants influence the waveguide's characteristic impedance, so that reflections take place at each discontinuity and separating layer respectively. A second very important requirement of the system is a high resolution. Thin layers $(<10 \mathrm{~mm})$ should be measured reliably. For that reason the pulse width must be sufficiently small. In this case a pulse width about $100 \mathrm{ps}$ is suitable. It is obvious, that a high bandwidth of the whole system is necessary to provide the precision and the resolution. One further requirement is a nearly jitter free generation of two pulse trains with slightly different pulse repetition rates. These pulse trains are used for sequential sampling. The following analog to digital conversion of the received signal occurs at a relatively slow rate, in order to allow an A/D conversion with a high resolution.
\end{abstract}

\section{Introduction}

There are many different concepts and techniques used in industrial level measurement applications like pulse radar, FMCW radar, systems with antennas or coaxial waveguides or systems with single rods or cables. Each concept has its advantages and its specific field of application. Here we seek for a high precision multi target level measurement system. The decisive parameters of such a system are explained in the following.

Correspondence to: M. Gerding

(michael.gerding@ruhr-uni-bochum.de)
One aim is to detect different layers of liquids in a tank. The desired goal is a resolution of less than $10 \mathrm{~mm}$. Furthermore, the achievable precision should be better than $1 \mathrm{~mm}$. These boundary conditions lead to a system with a comparatively high bandwidth of several GHz. Additionally, the system should be mechanically robust.

A solution is to combine the requirements of a wide-band signal generation with a suitable antenna or waveguide for a reasonable price. An initial step for this solution is the so called TDR principle (Time Domain Reflectometry). Electrical pulses of about 100 ps pulse duration are generated in the baseband. Therefore the hardware design is cost effective as compared to FMCW systems with a similar bandwidth. For the signal transmission, a TEM-waveguide is an optimum choice for a wide-band signal. Further positive aspects of such a waveguide are comparatively low losses and a very low dispersion. Furthermore the TEM-waveguide prevents any electromagnetic emission into the environment.

\section{A description of the measurement system}

The system can be divided into three main parts (see Fig. 1): the signal source, the sequential sampling unit and the coaxial waveguide within the tank. The function of the system can be summarized in the following way. If an input pulse encounters the discontinuity in the waveguide, caused by the different liquids dielectric parameters, the reflection travels back to the point of transmission where it is compared in time. The elapsed time is proportional to the liquid-level in the tank.

\subsection{The signal source}

As mentioned above, two pulse trains with slightly different pulse repetition rates have to be generated. Therefore, a crystal oscillator (XCO) with its constant frequency $f_{0}$ and a voltage controlled crystal oscillator (VXCO) with a slightly variable frequency are locked together by a PLL. The PLL provides a small frequency shift $\Delta f$ between the two crys- 


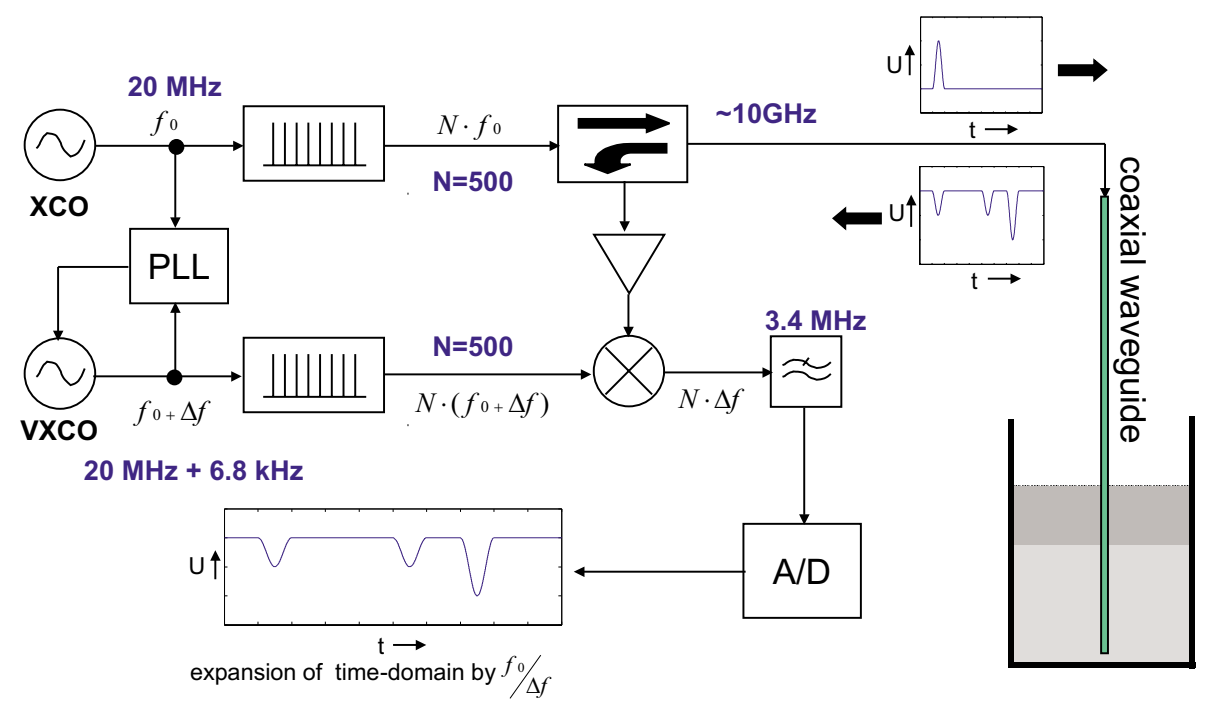

Fig. 1. Operating schematic diagram of the precision level measurement system (frequencies are only exemplary).

tal oscillator signals. For the given example the frequency shift is about $6.8 \mathrm{kHz}$. The phase noise requirements are very high. Crystal oscillators have a very good phase noise by themselves, so that the main attention has be payed to the PLL. A very small frequency shift can be obtained by using a special PLL concept while simultaneously using relatively small division ratios within the PLL, in order to keep a sufficiently low phase noise level.

The choice of the pulse repetition rate $f_{0}$ and the frequency shift $\Delta f$ depends on the application. The maximal length of the measuring probe determines the value of the pulse repetition rate, because the reflected pulses have to be unique in the observed time window and over the whole length of the probe respectively. Due to the expansion of the time domain by the factor $f_{0} / \Delta f$ the necessary acquisition time can be varied by the choice of $\Delta f$. Another limitation is the condition $N \cdot \Delta f<f_{0} / 2$ in order to avoid any image frequencies.

Each of the two sinusoidal signals is send to a comb generator where pulse trains are generated with a pulse duration of about $100 \mathrm{ps}$. This results in waveforms having a high harmonic content up to a bandwidth of around $10 \mathrm{GHz}$.

\subsection{The sequential sampling unit}

One of the wide-band signals is transmitted into the coaxial waveguide. At the points where the pulse encounters a discontinuity of the waveguide-impedance, caused by the liquid's surface, the pulse will be reflected. The reflected signal travels back to the point of transmission and is separated from the transmitted signal by a resistive coupler and forwarded to a sampling mixer. The sampling mixer is part of the sequential sampling unit, where the received signal and the slightly frequency shifted pulse train are mixed. By this technique, the two signals are slightly shifted against each other versus time. The complete waveform can be reconstructed in sequence after a complete acquisition cycle and the acquisition time $t_{a q}=1 / \Delta f$ respectively. Thereby the time domain is expanded by a factor of $f_{0} / \Delta f$. Looking at the frequency domain, the resulting mixer signal is the difference frequency between the transmitted signal frequency $N \cdot f_{0}$ and the slightly shifted signal $N \cdot\left(f_{0}+\Delta f\right)$. This results in the frequency $N \cdot \Delta f$, a low, easily handled frequency. The resulting low frequency signal is subsequently lowpass filtered and passed to a conventional analog to digital converter.

\subsection{The coaxial waveguide}

As can be seen from the diagram in Fig. 2, the waveguide is a coaxial waveguide which is electrically shorted at one end. The complete coaxial waveguide can be subdivided into the cable-to-waveguide connection, a short length of the waveguide for calibration, the so called "calibration line", and the actual measuring probe with a length of about three meters.

The reflections that occur within the waveguide are caused by a defined abrupt change of the inner conductor diameter or by a change of the waveguide impedance, caused by the liquids different dielectric constants. With the constant outer diameter $D$ the abrupt change of the inner diameter causes the reflection $r_{d}$ :

$r_{d}=\frac{\ln \frac{d_{1}}{d_{2}}}{\ln \frac{D^{2}}{d_{1} d_{2}}}$

This reflection is used for the calibration and as a reference distance respectively. The length of the calibration line with the diameter $d_{1}$ depends on the quality of the rfconnection. The better the adaptation into the waveguide, the shorter can be the length of this part. A poor matching of the rf-connection causes reflections and a ringing signal in the time domain, which may falsify the calibration impulse in the worst case. Therefore the calibration line must 


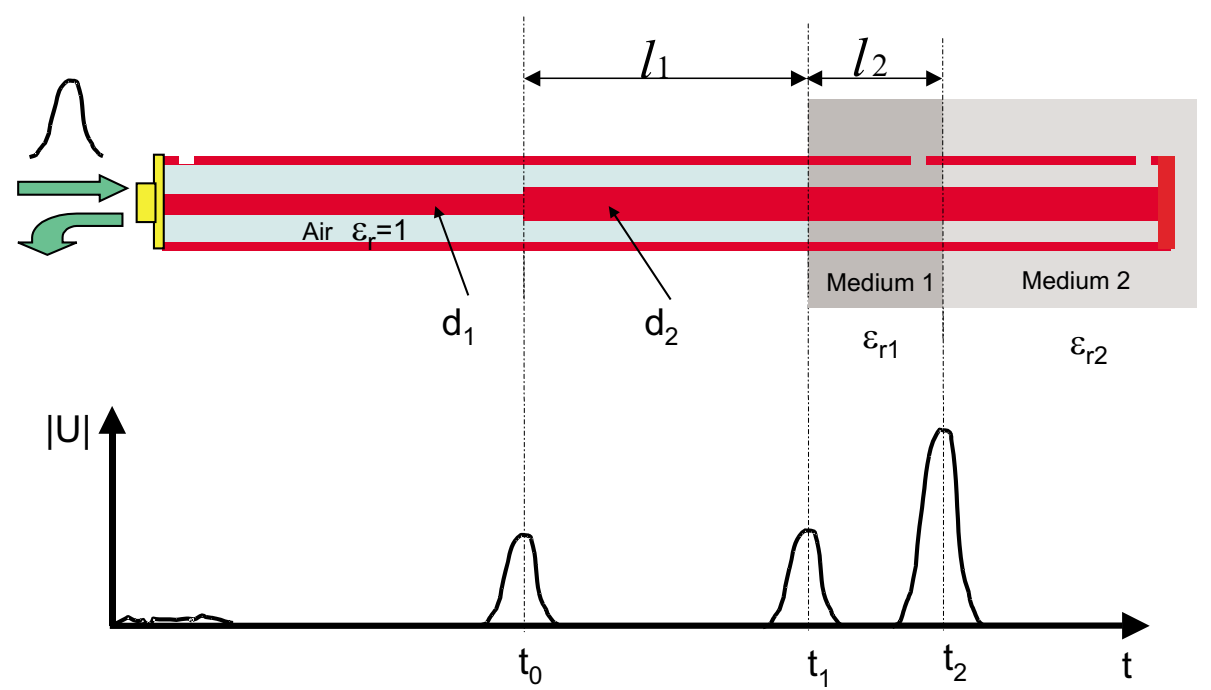

Fig. 2. Cutaway diagram of the coaxial waveguide and the corresponding received time domain signal.

be long enough for a complete decay of the pulse ringing. Beside a good wide-band matching, the rf-connection must be mechanically stable and robust, so that the inner conductor can be mechanically clamped between the two ends of the tube. This is important for a symmetrical positioning of the inner conductor in the center of the outer tube. Otherwise some undesired signal distortion can appear, caused by discontinuities of the distance between inner and outer conductor. Therefore a compromise must be found in the design of a mechanically robust and electrically well matched broadband rf-connection.

For the whole length of the measuring probe, the reflection coefficient $r_{f}$ is only determined by the dielectric parameters of the different fluids (Eq. 2).

$r_{f}=\frac{\sqrt{\varepsilon_{r 1}}-\sqrt{\varepsilon_{r 2}}}{\sqrt{\varepsilon_{r 1}}+\sqrt{\varepsilon_{r 2}}}$

For multi target level measurements, the liquids dielectric properties must fulfil a few requirements.

Firstly the upper liquid layer must not cause a total reflection, and secondly each reflection must be strong enough for a reliable detection. To minimize the attenuation of the penetrated liquid-layers, the liquids dielectric losses should be relatively small.

The holes along the outer tube allow the leakage and the inlet of air and the different fluids into and out of the tube respectively. They practically have no influence on the reflected pulses.

\section{Analysis of the time domain signals}

For an example of two different fluids in a tank, the time domain signal typically looks as shown in Fig. 2. The travel times of the transmitted pulses are representative for the fluids levels in the tank. To determine the fluid level, the timeof-arrival differences between the times $t_{1}, t_{2}$ and the time of the calibration pulse $t_{0}$ has to be calculated. With respect to Eq. (3), the distance $l_{1}$ is independent of any material parameter and can be obtained directly. In contrast to this, the reflection at the separating layer to medium 2 underlies a time distortion, caused by the dielectric constant of medium 1 . This implies the correction of the speed of light in Eq. (4) by the factor $1 / \sqrt{\varepsilon_{r 1}}$. The accuracy of the determined level $l_{2}$ directly depends on the knowledge of the dielectric constant of the penetrated medium.

$$
\begin{aligned}
& l 1=\frac{1}{2}\left(t_{1}-t_{0}\right) \cdot c_{0} \\
& l 2=\frac{1}{2}\left(t_{2}-t_{1}\right) \cdot \frac{c_{0}}{\sqrt{\varepsilon_{r 1}}}
\end{aligned}
$$

\section{Results of exemplary measurements}

A case study has been performed in order to evaluate the optimal system parameters like pulse width, bandwidth and transmitted power. A network analyzer is suited for this task, because all the mentioned parameters are easy to adjust and to change. In this case a HP8510B with a time domain option has been used. This means that the measured scattering parameter $S_{11}$ of the coaxial waveguide is transformed into the time domain by calculating an IFFT in order to determine the impulse response.The following measurements were obtained with the HP8510B and the following settings:

$\begin{array}{lc}\text { pulse width } & 50 \mathrm{ps} \\ \text { bandwidth } & 12 \mathrm{GHz} \\ \text { RF-transmitting power } & -10 \mathrm{dBm}\end{array}$

In the test fixture, the tank is filled with two different fluids: benzine $\left(\varepsilon_{r}=2.2\right)$ and water. The dielectric constant of water is a function of frequency, but for a first estimation this effect is neglected here and an average value of $\varepsilon_{r}=65$ 


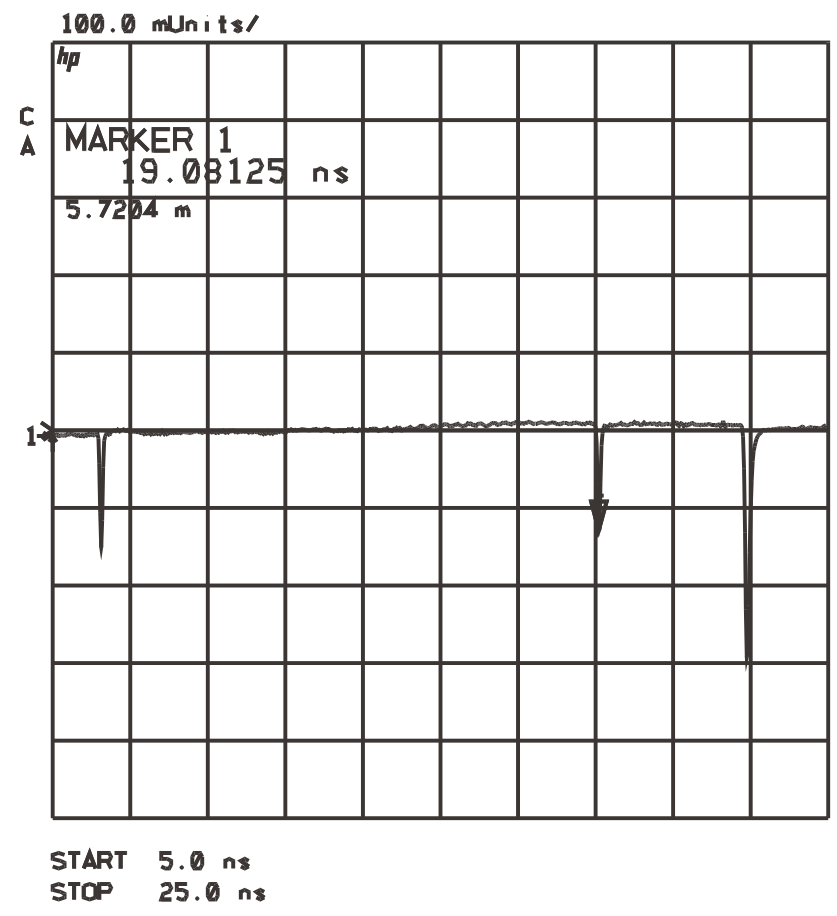

Fig. 3. Complete reflectogram: calibration impulse, reflections at the separating layers air-benzine and benzin-water.

is assumed for further calculations. The essential reason for the choice of these fluids is the very clear and abrupt separation layer between them, caused by their different specific density. The upper layer is benzine and the lower one water.

\subsection{The reflected time domain signal}

Figure 3 shows a complete reflectogram collected over the whole length of the measuring probe. With the knowledge of the liquids dielectric constants the reflection factor of each separating layer can be specified (air/benzine $-15 \mathrm{~dB}$, benzine/water $-3 \mathrm{~dB}$ ). Additionally, the attenuation of the coaxial waveguide has to be included into the considerations in order to interpret the plotted magnitude correctly. The attenuation of the coaxial waveguide filled with air is about $2 \mathrm{~dB} / \mathrm{m}$ and $7 \mathrm{~dB} / \mathrm{m}$ if it is filled with benzine. The reflection at the separating layer between benzine and water appears as a strong reflection, so that further separating layers would be difficult to detect.

The very first impulse on the left side of the diagram in Fig. 3 is the so called calibration impulse. This reflection is caused by the abrupt diameter change of the inner conductor (Eq. 1).

\subsection{Demonstration of the achievable precision}

Figure 4 demonstrates the achievable precision of the system. For this purpose the liquid's level has been changed by $0.665 \mathrm{~mm}$. As expected, the position of the impulse has obviously moved. The movement of the impulse is clearly

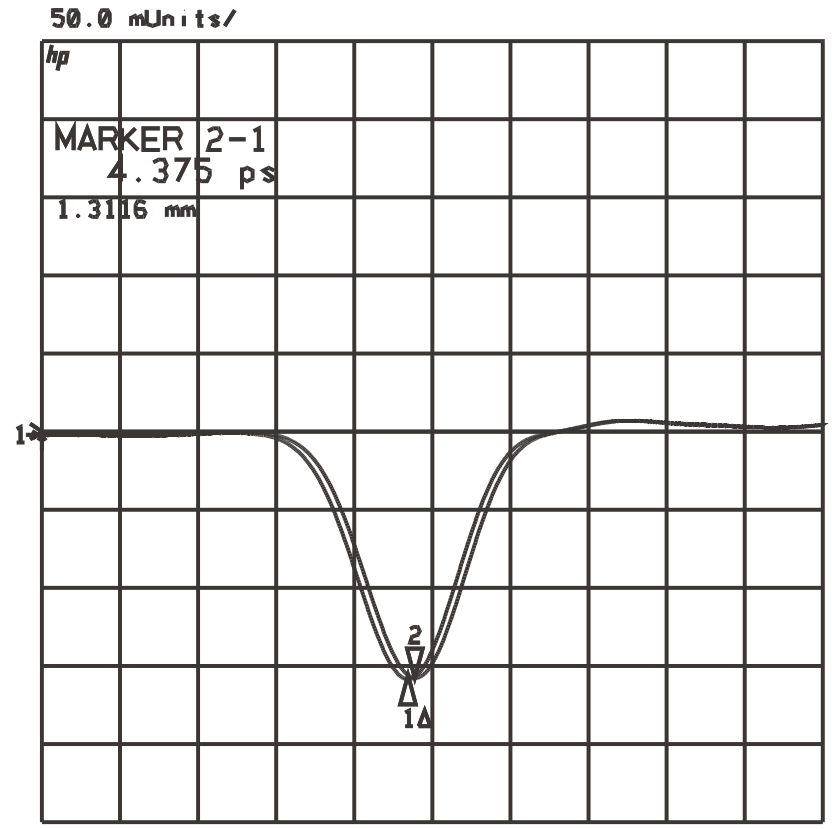

CENTER $9.038 \mathrm{~ns}$

SPAN $500.0 \mathrm{p}$

Fig. 4. Measured level variation of approximately $0.655 \mathrm{~mm}$.

visible, thus proving the ability to detect even smaller level variations with the help of mathematical algorithms.

\subsection{Demonstration of the achievable resolution}

In order to determine the achievable resolution of the measurement system, a thin layer of about $1 \mathrm{~cm}$ of benzine on water is characterized. As is shown in Fig. 5 the two reflected pulses can be separated clearly. If each pulse can be detected or separated as one single pulse and does not overlap with the other pulse, the detection of a layer is guaranteed. In order to separate layers thinner than $1 \mathrm{~cm}$, the pulse width can even be somewhat larger than it has been chosen for this measurement.

\section{Conclusion}

A mm-precision measurement system based on time-domain reflection has been presented in this paper. Beside the requirements on the system, the architecture of a prototype system and its functionality have been explained. Some exemplary measurement results, measured with a network analyzer, illustrate the functional principle.

Suitable system parameters (pulse duration, system bandwidth, transmitting power, pulse repetition rate) have been investigated, giving a basis for designing and optimizing the prototype system. The required pulse duration is about $100 \mathrm{ps}$ up to $150 \mathrm{ps}$. Comb generators based on step-recovery diodes (SRD) can fulfil these requirements. The technique is well known and the hardware complexity is moderate. The 


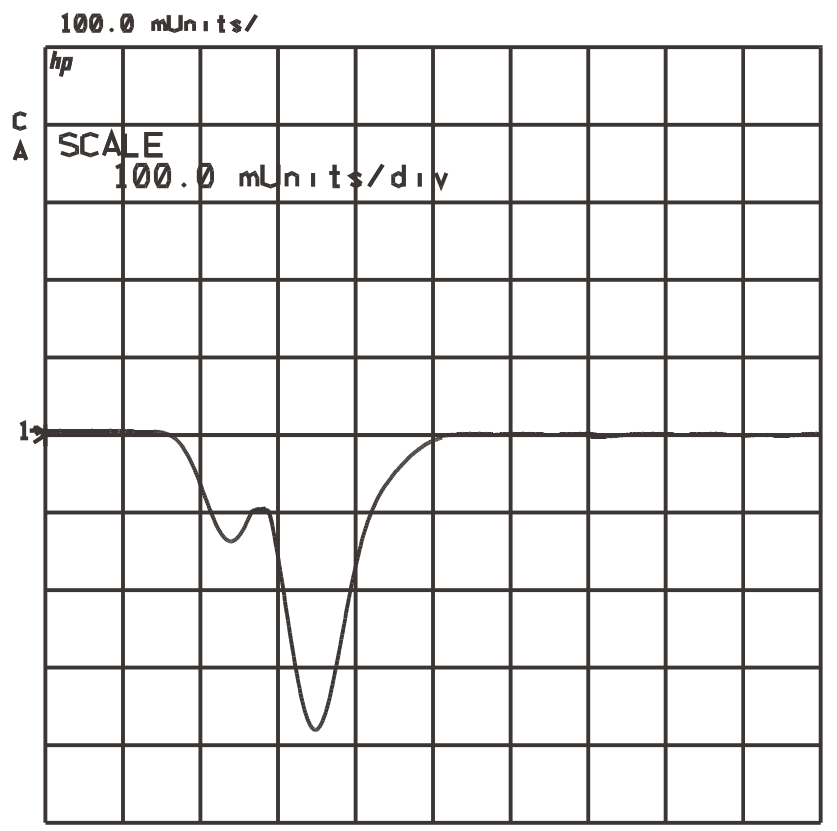

CENTER $19.9 \mathrm{~ns}$

SPAN $1.0 \mathrm{~ns}$

Fig. 5. Reflections at a layer with a thickness of about $1 \mathrm{~cm}$.

generation of two slightly frequency shifted pulse trains is the other principal item of the system and the next task to solve. The phase jitter of both signals must be as low as possible.

\section{References}

Hasted, J. B.: Aqueous Dielectrics, Chapman and Hall, 1973.

Heim, M.: Puls-Radar for mm-Precision in Level Measurement, Oldenbourg Verlag, 5/2000.

Schiek, B.: Grundlagen der Hochfrequenz-Messtechnik, Springer Verlag, 1999.

Hewlett Packard AN918 : Pulse and Waveform Generation with Step Recovery Diodes, http://rf.rfglobalnet.com/designcenters/ hp/lit/version.htm 